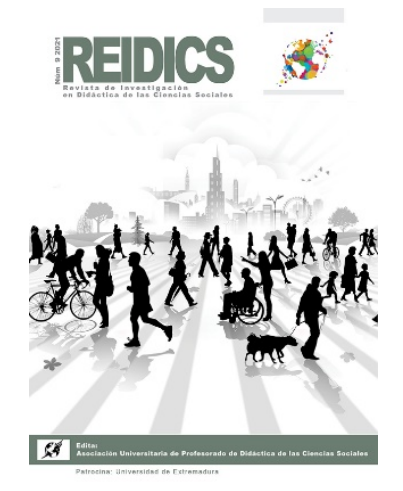

\title{
REIDICS
}

Revista de Investigación en

Núm. 9, 2021

Didáctica de las Ciencias

Sociales

Recibido 09 marzo de 2021

Aceptado 18 julio de 2021

E-ISSN: 2531-0968

\section{La Convención de los Derechos del Niño en las aulas}

\section{de Infantil}

Convention of the Rights of the Child in Early Childhood

\section{Classrooms}

Manuel José López Martínez

Universidad de Almería

Email:mlm138@ual.es

ORCID: https://orcid.org/0000-0001-6653-1398

DOI: https://doi.org/10.17398/2531-0968.09.238

\section{Resumen}

En el curso escolar 2019-20 tuvo resonancia mediática la celebración del 30 aniversario de la firma de la Convención de los Derechos del Niño (CDN) en 1989. Esta fue una de las razones para iniciar un trabajo reflexivo e investigador con el alumnado del Grado en Educación Infantil. Otra razón fue la detección de carencias en la formación adquirida a la hora de implementar el tratamiento didáctico del contenido de la Convención desde el enfoque de un conocimiento escolar social y crítico. El objeto de estudio se centra en conocer cómo se enseñan y aprenden estos derechos de la CDN en las aulas de la etapa de Infantil. Para ello, después de estudiar con cierta profundidad el significado de dicha Convención, se le propuso al alumnado que hiciera una micro-investigación etnográfica durante la fase del Practicum II con el fin de que observará y registrará cómo se desarrollaba la enseñanza del tiempo histórico. Este trabajo finalizaba con la elaboración de un informe individual en el que también se analizaba e interpretaba cómo se gestionaba dicha conmemoración en las aulas de Infantil. Los resultados obtenidos presentan una realidad diversa en los centros educativos cuando se encara el trabajo sobre la CDN.

Palabras clave: ciudadanía; derechos; infancia; formación inicial docente; investigación cualitativa.

\footnotetext{
Abstract

In the 2019-20 academic year, the celebration of the 30th anniversary of the Convention of the Rights of the Child (CRC) signed in 1989 had great media resonance. This was one of the reasons to start a reflective and research work with students of the Early Childhood Education Degree. Another reason was that shortcomings had been identified in the training acquired in previous academic years in implementing the didactic treatment of the Convention content from the point of view of social and critical educational knowledge. The
} 
object of the study is to learn how the 1989 CRC rights are taught and learned in the classrooms of the Early Childhood educational stage. For this purpose, after some in-depth study of the meaning of this Convention, a proposal to students was made to do a microethnographic research during the phase of Practicum II so that they could observe and record how the teaching of historical time was developed. This work ended with the preparation of an individual report that also analyzed and interpreted how the commemoration was managed in the Early Childhood classrooms. The results obtained present a diverse reality in educational institutions when faced with working with the CRC.

Keywords: citizenship; rights; childhood; initial teacher training; qualitative research.

\section{Introducción}

Si nos adentramos en la vida cotidiana de una escuela, es fácil observar en ella celebraciones o recordatorios de fechas y acontecimientos colectivos relevantes de la historia reciente. Ello responde, por una parte, a un componente cultural propio de las relaciones sociales en los diversos espacios públicos y privados y, por otra, a los tiempos de socialización establecidos de forma institucional para valorar las transformaciones experimentadas por los grupos humanos con la intención de asegurar una conciencia comunitaria. Celebrar significa reconocer un hecho que ha dejado huella en la sociedad, desarrollando para ello una serie de comportamientos y rituales en los que podemos apreciar ciertos consensos o imposiciones. En ese sentido, en los sistemas educativos públicos aparecen y reaparecen celebraciones debido a causas distintas. Así, la escuela, creada por los Estados-nación en el siglo XIX, fue utilizada para apuntalar el proceso de construcción de ciudadanos patriotas. Por esa razón la historia y la geografía "son disciplinas que han dado soporte a las identidades y memorias, individuales y colectivas" (Pérez Garzón, 2008, p. 39). Para Parra y Segarra (2012, p. 20) este tipo de celebraciones y actos en el ámbito escolar "tendían a la exaltación de las glorias nacionales". Hoy, a pesar de las transformaciones experimentadas, seguimos "limitando el conocimiento histórico escolar a una historia predominantemente política, protagonizada por varones y con una periodización eurocéntrica." (Ortega, 2020, p. 12). Sin embargo, creemos que la enseñanza de la historia debe ser "útil a los niños y niñas, a la juventud, para construir su historicidad y desarrollar su pensamiento y su conciencia histórica" (Pagès, 2019, p. 27).

La celebración de la ratificación por la Asamblea General de la ONU de la Convención de los derechos del niño y de la niña (en adelante CDN) el día 20 de noviembre de 1989 se ha convertido en una actividad visible en el calendario de las escuelas de nuestro entorno junto a otras, como son, por ejemplo, el día de la celebración del referéndum del 6 de diciembre de 1978 a favor del actual marco constitucional estatal, el día internacional de la Mujer o el día de la creación de cada comunidad autónoma. Sin embargo, se pueden apreciar diferencias sustanciales en cuanto al valor, la consideración y el espacio socioeducativo concedido a cada una de las celebraciones mencionadas, bien desde el punto de vista de cómo se organiza y se ejerce el poder para influir en la creación de identidades a escala regional o estatal, bien desde la transversalidad del valor universal de la igualdad de género como ejemplo de lucha y logro 
social. Todo esto obedece a cómo se manifiesta la microfísica del poder (Foucault, 2013) en las escuelas.

\section{Planteamiento del problema}

Sin duda, estas conmemoraciones en la educación formal presentan un valor pedagógico y didáctico. Se trata de actividades complementarias, organizadas en los centros educativos durante el horario escolar, que presentan diferencias con las consideradas propiamente lectivas. Sin embargo, su aprovechamiento como un contenido básico de la educación en valores democráticos no llega a explotarse al cien por cien en las diferentes etapas educativas. Es más, podemos avanzar como hipótesis de partida que, en repetidas ocasiones, debido al efecto de la costumbre, la rutina y de la tradición escolar, o de una insuficiente formación, no se aprecia esa dimensión de trascendencia que llevan implícitas estas acciones complementarias para reconocer el derecho y las obligaciones de niños, niñas, adolescentes y jóvenes como actores protagonistas que pueden revitalizar el funcionamiento de la democracia. Desde nuestra óptica, ha de prevalecer una enseñanza y aprendizaje de las Ciencias Sociales (en adelante CC.SS.) en las infancias y en la juventud en la línea de cumplir una función social que refuerce el funcionamiento de la democracia (Gutiérrez y Pagès, 2018) y de los Estados de derecho en un contexto condicionado por la globalización de un modelo de vida, anclado éste en la hegemonía de los valores individualistas de la ideología neoliberal capitalista.

En el curso escolar 2019-20 se celebró el 30 aniversario de la firma de la CDN, un hito en la historia reciente comparable con la significación histórica de la caída del Muro de Berlín. Esta fue una de las razones para iniciar un trabajo reflexivo e investigador con el alumnado del Grado en Educación Infantil (en adelante EI). Otra razón fue la de haber detectado en dicho alumnado ciertas carencias formativas a la hora de implementar el tratamiento didáctico del contenido de la Convención desde el enfoque de un conocimiento escolar social y crítico. El objeto de estudio se centra, pues, en conocer cómo se enseñan y aprenden estos derechos de la CDN en las aulas de Infantil. Para ello, después de estudiar su significado desde la didáctica de las CC.SS., se pidió al alumnado que hiciera una micro-investigación etnográfica durante la fase del Practicum II. El propósito consistía en que observará y registrará cómo se desarrollaba la enseñanza del tiempo histórico en las aulas de Infantil. Este trabajo finalizaba con la elaboración de un informe individual en el que, entre otras tareas, se analizaba e interpretaba dicha conmemoración desde la perspectiva de la enseñanza y el aprendizaje del tiempo histórico en Infantil.

Esta iniciativa enlaza con las recientes reflexiones de Carretero (2019) sobre el aprendizaje de la historia, al considerar este autor que "las celebraciones históricas escolares pueden y deben ser objeto de una reflexión compleja porque ponen en cuestión la cantidad y calidad de la historia que se aprende en la escuela" (Carretero, 2019, p. 5). Por nuestra parte, nos preguntábamos por la calidad de la ciudadanía enseñada y aprendida en los centros educativos a través de esas celebraciones escolares. En esta línea, el trabajo de Parra y Segarra (2012) en el contexto de la Comunidad Valenciana sobre cómo se desarrollan dos fechas destacadas en centros de Primaria, el 9 de octubre, día de la Comunitat Valenciana y el del referéndum de la Constitución del 6 de diciembre de 1978, concluye que el tratamiento escolar plantea escasas 
conexiones con una educación ciudadana más abierta, reflexiva, crítica e intercultural. Según estos autores, una de las razones posibles de esta realidad estriba en el "filtraje" para eliminar "los componentes políticos que puedan suscitar polémicas, y que, desgraciadamente, conduce a una educación ciudadana muy pobre" (Parra y Segarra, 2012, pp. 31-32). Teniendo en cuenta lo anterior, se nos planteaba la duda de si estas circunstancias se podrían repetir en las aulas de Infantil con la celebración de la CDN.

\section{Fundamentación teórica}

La CDN es el tratado de derechos humanos que ha logrado un mayor compromiso internacional. La visibilización de esta efeméride en la escuela refleja una mayor sensibilización hacia el papel que representan las infancias desde una perspectiva social y política, con una orientación más activa, más justa y equitativa en los estados denominados democráticos. Formalmente, queda así superada una concepción hegemónica y adultocéntrica que concibe a niños, niñas y jóvenes como seres no completos y en fase de tránsito hacia la situación ideal, la adulta, esperada en el imaginario colectivo. Por tanto, la participación infantil se sitúa mayoritariamente en espacios controlados por los modelos hegemónicos paternalistas y proteccionistas (Lay-Lisboa y Montañés, 2018) que evidencian la subordinación de las infancias hacia el poder ejercido por los adultos, a pesar de las posibilidades que se abren en los niveles más altos de la escalera de la participación de Hart (1993).

No obstante, como sostienen Bruck y Ben-Arieh (2020), en los últimos años se han producido una serie de cambios en las concepciones y miradas hacia las infancias teniendo en cuenta la firma de la Convención. Para estos autores:

(...), ha habido algunos avances importantes en la comprensión y el estudio del bienestar de los niños y niñas, pasando del welfare (estar bien) al well-being (sentirse bien) (Kamerman et al., 2009), y de la protección de la infancia al desarrollo del niño (Khan, 2009). (Bruck y Ben-Arieh, 2020, p. 36).

Observando los datos, niños y niñas y adolescentes, comprendidos entre los 0 y 17 años, suponen un poco más del 30\% de la población mundial (Sanz Gimeno, 2020, p. 6). Según este autor, si nos fijamos en las fases vitales por las que pasa este sector social, se distinguen tres grandes etapas:

\section{Tabla 1}

\begin{tabular}{lr}
\hline La primera infancia (de 0-6 años) & $11 \%$ \\
La etapa escolar y pre-adolescente (entre los 6 y 11 años) & $10 \%$ \\
La adolescencia (entre 12 y 17 años) & $10 \%$
\end{tabular}

Fuente: elaboración propia a partir de Sanz Gimeno, 2020, p. 9

Por tanto, el logro más destacado o el ideal de estos esfuerzos en el campo educativo formal residiría en la implementación de los contenidos de esta Convención en la vida, organización y funcionamiento diario de un centro educativo. Tenemos ejemplos de esfuerzos por convertir los artículos de la CDN en ejes vertebradores no solo de la vida de una comunidad escolar sino también del espacio urbano. Una muestra está representada, por ejemplo, en el movimiento de ciudades educadoras iniciado en Barcelona en los años 90, en el trabajo llevado a 
cabo por Tonucci (2009), que ha cristalizado en la red de ciudades de los niños, en la creación de las Ciudades Amigas de la Infancia, en el proyecto "Nosotros proponemos" iniciado en Portugal en 2011 (Rodríguez y Claudino, 2018), o también en las experiencias educativas sobre participación ciudadana desarrolladas en Europa, como es el caso del proyecto STEP con profesorado de infantil y primaria en Italia, Francia y España (Estepa y García Pérez, 2020). En nuestro país, y con un recorrido temporal menor, resaltamos los esfuerzos para la obtención del reconocimiento como centro referente en educación en derechos de infancia y ciudadanía global establecidas por UNICEF Educación (2019).

Estas propuestas asumen el derecho de ciudadanía activa de niños, niñas y jóvenes a participar y a ser escuchados por los adultos en la toma de decisiones en el espacio público. En nuestro país resaltamos la aportación de Llena y Novella (2018) en cuanto a la participación infantil y los consejos de infancia. Igualmente, tenemos conocimiento suficiente de los beneficios que provocan la incorporación de los derechos de la infancia y la ciudadanía global en las instituciones escolares. Según Urrea et al. (2018), se han evaluado programas en Canadá (Rights, Respect and Responsability (RRR) de 2011) y en Inglaterra (Rights Respecting Schools Award (RRSA) de 2008), en los que se aprecian estas consecuencias positivas. Con su desarrollo se obtienen grandes beneficios para el conjunto de la comunidad educativa, destacando:

(1) Mejora de la autoestima del alumnado; (2) Reducción de los prejuicios; (3) Mejora de las conductas y las relaciones entre los miembros del centro; (4) Satisfacción entre el profesorado; (5) Mejora de los resultados académicos; y (6) Reconocimiento de todos los miembros de la comunidad (Sebba y Robinson, 2010). (Urrea et al., 2018, p. 130).

Sin embargo, a pesar de estos avances, se comprueba que los artículos de la CDN no se mencionan en los desarrollos curriculares de la educación obligatoria (Jerome et al., 2015, citado por Urrea et al., 2018). No aparece una sola mención expresa a los artículos de la Convención de 1989 si analizamos el contenido de los principios generales, los fines y los objetivos de la Orden ECI/3960/2007, de 19 de diciembre, por la que se establece el currículo y se regula la ordenación de la educación Infantil a nivel estatal en España. Lo mismo ocurre en sus 8 artículos con la Orden del 5 de agosto de 2008 por la que se desarrolla el currículo correspondiente a la Educación Infantil en la comunidad autónoma de Andalucía. Si bien es cierto que en algunos apartados se alude a los derechos de la infancia, sin embargo, no se explicita claramente la CDN de 1989. Se requiere de una lectura detenida entre líneas para detectar los derechos de la primera infancia recogidos en la normativa educativa vigente. Esto evidencia una carencia en la representación social de este hito histórico en el marco educativo. Será solo recientemente, con la aprobación en nuestro país de la Ley Orgánica 3/2020, de 29 de diciembre, (LOMLOE), cuando se incluya expresamente como principio rector del sistema educativo la mención a esta normativa internacional que fue aprobada ampliamente por la Asamblea General de la ONU el 20 de noviembre de 1989. Recordemos que en España entró en vigor el 5 de enero de 1991, es decir, hace más de 30 años. 


\subsection{El derecho a la participación infantil}

De todo lo anterior se desprende que fortalecer la participación infantil es una prioridad, pero falta la parte esencial y es que sea real (Novella y Trilla, 2014). Dicho esto, reivindicamos una participación de aquellos sectores sociales minoritarios en una sociedad adultocéntrica hegemónica (Gaitán, 2018), sabiendo que existen resistencias culturales para una intervención infantil en la toma de decisiones políticas, a pesar de las exigencias de la Convención y de la obligatoriedad de los Estados firmantes a cumplir este tratado internacional. Por estas razones, el o la futura docente novel de Infantil que se está formando en la Universidad tiene que tomar conciencia de los distintos niveles de actuación que se puede encontrar en las escuelas, encaminados a la consecución de esa mayor sensibilidad institucional hacia las infancias para incidir en una socialización política más justa y equilibrada. Dicha socialización requiere de un escenario cotidiano para desarrollarla ampliamente en función del modelo de ciudadanía que se pretende alcanzar. En el desempeño de su labor docente han de incluirse acciones encaminadas al fomento de la participación infantil en el espacio público escolar y comunitario mediante el conocimiento y el cultivo didáctico del contenido de la CDN.

Desde ese punto de vista, la propuesta formativa desarrollada en la asignatura Aprendizaje de las CC.SS. en Educación Infantil (9 ECTS) con el alumnado de $3^{\circ}$ del Grado en EI se ha centrado en sensibilizar a este estudiantado sobre las posibilidades educativas que presenta el trabajo con la CDN. Se trata de un recurso didáctico para incentivar una práctica docente desde una perspectiva crítica y transformadora de la educación. Como formadores de futuros docentes de EI y desde un planteamiento crítico de la Didáctica de las CC.SS., creemos en la necesidad de ofrecer al alumnado universitario una visión amplia del conocimiento social escolar (Orden ECI/3854/2007), de carácter emancipador, para que pueda actuar en una realidad caracterizada por la complejidad (Morin, 2001) y la desigualdad. En este sentido, una de las competencias exigidas al estudiante universitario de este Grado es la social y ciudadanía global (Competencias transversales de la Universidad de Almería aprobadas en Consejo de Gobierno, 17 de junio de 2008), sustentada en la reflexión crítica sobre el conocimiento para que se consigan mayores cotas de justicia social en el conjunto de la comunidad. Aunque este alumnado recibe una formación adaptada a su campo de actuación, como novedad, integramos en nuestra asignatura marcos de conocimiento procedentes de las diversas CC.SS. que incluyen contenidos y espacios que no han estado presentes en las tradiciones académicas, como es el caso de las aportaciones de la nueva sociología de la infancia. En esta línea, Gaitán (2006) muestra el origen de esta subdisciplina sociológica y en la que se exploran nuevos campos para indagar con otra mirada la realidad diversa y compleja que envuelve a las infancias teniendo presente tres objetivos básicos:

a) contribuir al crecimiento de las ciencias sociales en general, incorporando la visión de uno de los grupos componentes de la sociedad, a menudo olvidado; b) aportar explicaciones sociológicas en el necesario enfoque interdisciplinar de un fenómeno complejo como es la infancia; c) dar visibilidad a los niños como actores sociales, en línea con las orientaciones de la Convención Internacional que reconoce a los niños y niñas como sujetos de derechos. (Gaitán, 2006, 10). 
Es sabido que cualquier selección de contenidos curriculares responde a una manera de mirar la realidad. Sin embargo, tenemos a nuestro favor que el desarrollo curricular vigente en la etapa de EI se plantea desde un enfoque globalizador entretejiendo diversas áreas de conocimiento. Centrándonos en nuestra labor formadora con un estudiantado que mayoritariamente desarrollará sus competencias y saberes en un escenario escolar, es preciso que se tenga en cuenta ese enfoque, amparado en la reflexión crítica sobre la práctica docente, desde el reconocimiento de la observación como técnica esencial de la investigación educativa etnográfica. En nuestros días, un buen docente ha de investigar, reflexionar e innovar en y sobre su práctica docente.

\section{Estrategia formativa y metodología de la investigación}

Es evidente que la formación inicial del profesorado ha de incidir en los procesos de pensamiento, creencias y concepciones pedagógicas de los futuros maestros, ya que en numerosas ocasiones están muy arraigadas antes de decidir ser docente (Porlán, Rivero y Martín del Pozo, 1998). Por ello, dependiendo de cómo se construya el entorno de aprendizaje en la formación inicial, así será la toma de conciencia sobre las posibilidades que ofrecen los espacios públicos para la enseñanza del conocimiento social. Así, se inició un ejercicio reflexivo conjunto entre este formador y su alumnado sobre la práctica docente desde el campo de la didáctica de las CC.SS., aprovechando situaciones reales, no solo en el aula universitaria, sino también en las experiencias de inmersión profesional adquiridas en la fase de Practicum.

En el curso académico 2019-20 propusimos al alumnado de dos grupos de 3ํㅜ $(\mathrm{N}=139,131$ mujeres y 8 varones) de la asignatura anual Aprendizaje de las Ciencias Sociales en Educación Infantil que fijara su atención en sus concepciones implícitas sobre la participación infantil en la toma de decisiones en los espacios públicos, con la finalidad de trasladarlas de manera explícita a otras más complejas. Este fue el punto de arranque de la investigación que aquí exponemos. En este estudio cualitativo en el aula universitaria, desde la didáctica de las CC.SS. partimos de la pregunta siguiente: ¿cómo podemos mejorar la formación inicial del futuro docente para que incida positivamente en la educación integral de la ciudadanía infantil, desde el conocimiento y aprovechamiento de los contenidos de la CDN de 1989?

Teniendo en cuenta el contexto en el que nos situamos, damos respuesta desde el enfoque de una educación para una ciudadanía democrática en el segundo ciclo de EI, sabiendo además que el municipio donde residimos había solicitado el reconocimiento formal como Ciudad Amiga de la Infancia para el período 2018-2022. Este cambio en la estrategia formativa en esta asignatura del Grado se originó después de haber detectado carencias mientras la impartíamos en cursos anteriores, concretamente referidas a la manera de tratar didácticamente el contenido de la Convención en EI. Por tanto, en un principio han sido dos las acciones innovadoras incorporadas al proceso formativo siguiendo el modelo de la investigación-acción en esta asignatura. Una primera, cuyo fin era atender y cubrir el vacío comprobado sobre la significación histórica de la Convención, promoviendo en el estudiantado universitario miradas de largo alcance, superando la hegemónica interpretación legalista de los Derechos del niño. Una segunda acción, en la fase del Practicum II, invitando al alumnado en formación a la realización de una 
micro-investigación etnográfica individual con el fin de conocer cómo se trabaja el tiempo histórico en las aulas de Infantil a partir, entre otros contenidos, de la celebración del día de los Derechos del Niño y de la Niña.

Como formadores de futuros docentes, creemos en la necesidad de mostrar una visión amplia del conocimiento escolar de carácter emancipador. En este sentido, una de las competencias exigidas al estudiante universitario es la social y ciudadana, sustentada en la reflexión sobre el conocimiento escolar para conseguir mayores cotas de justicia social. Creemos que las aulas universitarias son un espacio propicio para la reflexión y la crítica de la realidad social, utilizando para ello a las CC.SS. con la finalidad de incentivar la acción colectiva para cuidar el buen funcionamiento de la democracia (Pagès, 2019). Sostenemos, pues, desde ahí, que la selección de contenidos y la construcción de experiencias de enseñanza y aprendizaje merecen ser repensadas en el discurrir diario de la práctica docente.

Como consecuencia de todo lo anterior, en nuestra asignatura se prestó una especial atención a la hora de iniciar el estudio del contenido de dicha Convención. Estos futuros profesionales noveles están obligados a reconocer las capacidades y posibilidades de sus infantes como ciudadanos activos, con derechos y deberes, capacitados para contribuir a la construcción de la vida social presente, y no como meros actores pasivos, incompletos, que se preparan para el futuro, una imagen, por cierto, creada por la mirada adulta que persiste a lo largo del tiempo. En esa línea, Hoyuelos y Riera (2015) señalan que la construcción de la personalidad depende de cómo el otro o la otra "nos ve, nos aprecia y nos considera" (p.15). Sin este reconocimiento expreso, claro y nítido del futuro docente, es más fácil que la formación integral de niños y niñas de 0-6 se resienta debido a la presión de una mirada jerarquizada adultocéntrica (Gaitán, 2018). De ahí la exigencia de estudiar con cierto nivel de profundidad la CDN como eje educativo en la etapa de Infantil, al mismo tiempo que se va construyendo la identidad docente en la formación inicial al comprometerse con la igualdad intergeneracional y con la comunidad social en la que desempeñará su trabajo. Hemos pretendido con esta experiencia formativa que el estudiantado advierta la influencia de los diferentes contextos en el proceso de enseñanza y aprendizaje en el aula. Desde esta orientación, se requiere una formación amplia sobre el significado histórico de la aprobación de la CDN de 1989, con la finalidad de favorecer la formación integral de las criaturas como ciudadanos activos. Si se llegara a interiorizar esta renovada mirada, se estarían dando pasos para reconocer que estas edades contribuyen a la construcción de la sociedad democrática del presente.

\section{Plan de acción. Fases, contenidos formativos y acciones investigadoras}

Teniendo en cuenta el objeto de estudio, se ha desarrollado una metodología activa, trabajando esta temática de forma individual y por grupos de trabajo, donde se ha pensado en el significado educativo de la CDN de 1989 y las posibles aplicaciones en el desarrollo curricular vigente de la etapa de Infantil, especialmente en el segundo ciclo. Por tanto, se han planteado distintas fases atendiendo a las cuestiones referidas a qué sabemos, qué queremos saber y cómo lo vamos a realizar, siguiendo el método de proyectos de trabajo y de la investigación en la acción. En un principio, han sido dos las acciones innovadoras incorporadas al proceso 
formativo siguiendo el modelo de la investigación-acción. Una primera para cubrir el vacío detectado sobre la significación histórica de la Convención en paralelo a la caída del Muro de Berlín de 1989, promoviendo miradas de largo alcance, superando la interpretación legalista y exclusivamente proteccionista de los Derechos del niño. Una segunda acción, en la fase del Practicum II, invitando al alumnado en formación a la realización de una micro-investigación educativa cualitativa etnográfica individual, reflejada en un informe final escrito mediante un trabajo de campo desarrollado desde el 18 de noviembre de 2019 al 17 de enero de 2020, usando la técnica de la observación participante con el fin de describir de forma natural cómo se trabaja el tiempo histórico en las aulas de EI, prestando especial atención a las actividades desarrolladas con motivo de la celebración el 20 de noviembre del día de los Derechos del niño y de la niña. Conociendo la relevancia que presenta el Practicum para el alumnado universitario, con esta propuesta de investigación realizábamos una labor de reconocimiento para demostrar que los estudiantes son sujetos epistémicos (Rivero y Porlán, 2017), capacitados para elaborar conocimiento valioso sobre la práctica docente.

Fase 1. Actuaciones antes del Practicum

En esta fase inicial del curso, además de comenzar un proceso de interiorización de las finalidades y los conceptos clave de la enseñanza de las CC.SS., quisimos que el alumnado revisará sus concepciones sobre la enseñanza de la Historia y del tiempo histórico. Así, debía recordar cómo fueron sus experiencias en su etapa preuniversitaria para tomar conciencia del modelo de enseñanza y aprendizaje que había vivido de la Historia en etapas escolares anteriores, tanto en la escuela como en el instituto. En esa línea, para que pudiera contrastar esa experiencia personal y formando parte de los diferentes grupos de trabajo, se le propuso el análisis de una experiencia innovadora realizada por docentes de Infantil en un colegio público (Pérez, Baeza y Miralles, 2008). Desde aquí, se trazó una línea de actuación formativa para resaltar la importancia de seleccionar contenidos de la vida cotidiana en cualquier contexto escolar que pudieran propiciar el aprovechamiento del principio de temporalidad, eje central de cualquier pensamiento histórico. Se le planteaba la oportunidad de un aprendizaje que nacía con las celebraciones del calendario escolar. Se mostraba al alumnado del Grado cómo se podía conectar pasado, presente y futuro para que fuesen enseñados y aprendidos en la etapa de Infantil. Dentro de las diferentes celebraciones incluidas en el calendario escolar se seleccionó como ejemplo ilustrativo la del 20 de noviembre puesto que era una fecha en la que el estudiantado estaría realizando su fase de prácticas externas en los centros educativos desde el 18 de noviembre de 2019 hasta el 17 de enero de 2020.

Situar el foco de atención en esta celebración obedece a una razón: la de haber detectado carencias en el conocimiento del alumnado universitario. A lo largo de estos años de docencia hemos podido comprobar que la mayor parte del alumnado tiene un conocimiento superficial sobre la trascendencia histórica de la ratificación de la Convención por la Asamblea General de Naciones Unidas en 1989. Se tiene un conocimiento mínimo, adquirido en los ciclos formativos de Técnico Superior y en alguna asignatura de la formación inicial. No se ha forjado con garantías una conciencia histórica sobre el significado de esta Convención. De ahí que se proceda a ampliar el volumen de contenidos conceptuales, procedimentales y actitudinales sobre la CDN 
de 1989 para cristalizar sus competencias, enlazando teoría y práctica con el fin de llevarlas a un aula de Infantil. En la primera acción se ofreció una panorámica histórica del proceso seguido hasta la firma de la Convención en 1989, destacando varios momentos: la explicación de la inexistencia de Derechos en el siglo XIX por diferentes razones, la creación de Save the Children, la contribución de Janusz Korczak, el pedagogo y médico polaco que "por primera vez formuló una concepción emancipadora de sus derechos" (Liebel, 2019, 178), la Declaración de Ginebra sobre los Derechos del Niño de 1924 y la Declaración de los derechos del niño de 1959.

Una vez mostrado el panorama y el contexto previo en el que se aprobó la Convención se procedió a la elaboración de unidades didácticas integradas para trabajar el 20 de noviembre como oportunidad de aprendizaje desde las tres áreas de conocimiento del desarrollo curricular vigente de Infantil en la Comunidad Autónoma de Andalucía (Orden del 5 de agosto de 2008): conocimiento de sí mismo y autonomía personal, conocimiento del entorno y lenguaje, comunicación y representación. El motivo principal fue la celebración del 30 cumpleaños de la Convención presentando una tarta en el aula de Infantil como banderín de enganche para suscitar el interés del alumnado de segundo ciclo de Infantil. De esta manera, se utilizaba un cumpleaños como disparador del proceso de enseñanza y aprendizaje, sabiendo que el alumnado infantil presta una gran atención a estos acontecimientos cotidianos. Se vio conveniente que el alumnado universitario trabajara en la elaboración de un mapa conceptual donde se integraran las finalidades de la enseñanza de las CC.SS., los conceptos clave de las CC.SS., las diferentes CC.SS., el desarrollo curricular vigente y las tres áreas de conocimiento con sus bloques de contenidos, y todo ello desde el marco de un modelo alternativo de enseñanza de las CC.SS. (Galindo, 2016), un mapa planteado para desarrollar el pensamiento histórico en el segundo ciclo de Infantil. Con este trabajo previo de formación, el estudiantado universitario había tomado conciencia de la trascendencia educativa de la Convención, y estaba preparado para comprobar la dimensión real de su aprovechamiento educativo en las aulas de Infantil durante el período del Practicum.

Fase 2. Durante el Practicum

Antes de comenzar este periodo formativo, el alumnado recibió una serie de consignas para realizar una observación participante con el fin de comprobar si el 20 de noviembre se celebraba o no en las escuelas y cómo se llevaban a cabo esas actividades complementarias, teniendo presente que se trataba de un contenido educativo de notable relevancia para trabajar el tiempo histórico y la Historia en la etapa de EI. Coincidimos con Riera (2015) en la utilidad de la observación como un componente esencial de la reflexión sobre la práctica docente. Para esta autora, "La observación y el análisis de lo observado debería servirnos para plantear cambios en el diseño de las propuestas educativas, regularlas y mejorarlas (...)" (Riera, 2015, p. 63). Pero la observación no cumple su finalidad si no se registra lo observado y se interpreta. Por esa razón, el estudiantado del Grado tenía que realizar y entregar un informe individual de lo observado en el aula y en el centro en su período de Practicum II sobre el tratamiento de la celebración del 20 $\mathrm{N}$ dentro de una micro-investigación más amplia acerca del tiempo histórico en Infantil. Estamos convencidos de que las prácticas externas pueden ser un contexto óptimo para transformar las concepciones de los futuros maestros y maestras relativas a la enseñanza y aprendizaje de las 
ciencias sociales (López, Felices, Jiménez y Moreno, 2018). En ese recorrido es importante acompañar en su propia práctica al estudiante que se está formando y orientarle en el contexto profesional en que interviene.

Como hemos indicado, al estudiante se le ofreció una serie de consignas que a continuación se describen, para que pudiera registrar la observación:

\section{Figura 1}

Dimensiones recomendadas para realizar el registro de la observación en el aula de Infantil

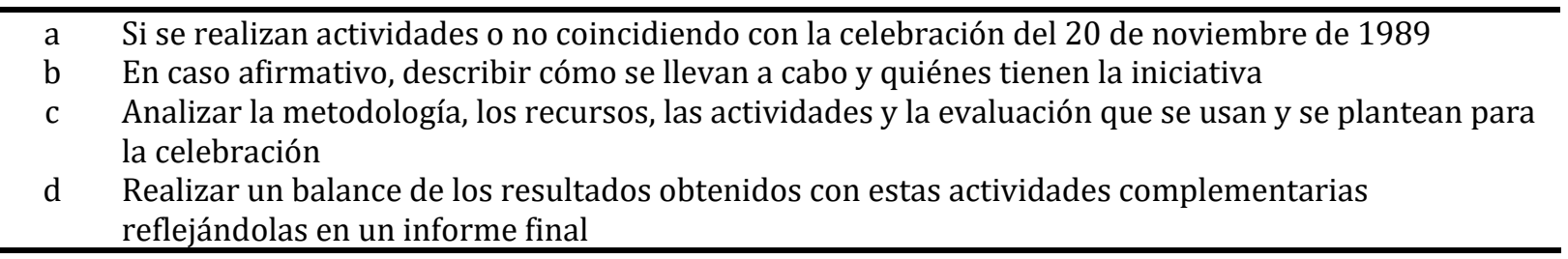

Fuente: elaboración propia

Fase 3. Actuaciones a posteriori del Practicum.

Tras la indagación, el alumnado elaboró un informe final sobre la micro investigación etnográfica realizada en el aula acerca de la enseñanza de la historia en Infantil. En dicho informe se describen y se analizan de forma reflexiva diversas situaciones detectadas en el aula y en el colegio sobre la enseñanza del tiempo histórico recurriendo a distintas situaciones del proceso de enseñanza y aprendizaje. Igualmente, se hace mención a la celebración de la Convención, indicando cómo se habían desarrollado esas actividades por parte del tutor o tutora y cómo las enfocarían ellos y ellas, teniendo en cuenta la formación adquirida en la asignatura Aprendizaje de las CC.SS. en EI. Finalizado el Practicum II, se analizó el contenido de los informes a partir de las 4 dimensiones expuestas anteriormente en el Cuadro 1 sobre la experiencia relacionada con la enseñanza del tiempo y el pensamiento históricos en la escuela, teniendo como centro de interés la celebración de la CDN.

\section{Resultados de la micro-investigación realizada por el alumnado}

Analizados los contenidos de 124 informes del curso académico 2019-20, sobre un total de 139 personas matriculadas oficialmente en nuestra asignatura, los resultados obtenidos presentan una realidad diversa en los centros educativos y en las aulas de Infantil cuando se encara el trabajo con la CDN vinculándolo con la enseñanza y aprendizaje del tiempo histórico. Si hacemos un balance global, podemos decir que, en la mayoría, es decir, en 77 de ellos, que se corresponde con un poco más del $62 \%$ de los centros donde nuestro alumnado ha estado realizando el Prácticum II, se conmemora este acontecimiento histórico. Sin embargo, no se menciona esta efeméride en 47 de los informes entregados. Se comprueba así que mayoritariamente sí se llevan a cabo diversas actividades para celebrar la ocasión, aunque un aspecto a resaltar es la gradación de situaciones educativas con las que se encontró el alumnado del Grado en Educación Infantil.

Podemos detectar casos en donde no existe celebración y, por tanto, la no realización de actividades de acompañamiento en las aulas, tanto en centros concertados como públicos, 
siendo este porcentaje de un 38\%. Otros centros hacen una tratamiento efímero durante un tiempo muy reducido por parte de la docente-tutor/a, pasando por otros colegios en donde sí se realizan actividades a lo largo del día del $20 \mathrm{~N}$ en los que el conjunto del profesorado del ciclo de Infantil participa con la implicación de la dirección del centro y de las familias, llegándose a detectar casos en los que se planifica un trabajo previo, desarrollado ampliamente a lo largo de la semana de dicha fecha, siendo minoritario este último planteamiento didáctico en el conjunto de centros investigados. Por la información suministrada por el alumnado universitario, se comprueba la paradoja de que en un mismo centro existen diferencias notables en el tratamiento de este tema, no sólo entre las etapas educativas, sino también entre el profesorado de un mismo ciclo, por ejemplo, de 3 a 5 años, demostrándose así la dependencia del nivel de concienciación y conocimiento sobre la temática por parte del equipo docente responsable.

El patrón de actuación comprobado en la secuenciación de actividades por la mayoría del alumnado del Grado ha sido el siguiente. En primer lugar, se pregunta y se habla en la asamblea sobre esta temática para ver qué conocen los niños/as, estableciéndose comunicación y turnos de palabra después de que la docente expone de forma oral la importancia de ese día. A continuación, se muestra un vídeo sobre los Derechos de los Niños con distintos personajes infantiles que aparecen en los medios de comunicación, realizando seguidamente una ficha o una representación sobre los derechos para llevarla a casa. Hay ejemplos en los que se recrea un corazón en una cartulina donde escriben 3 derechos fundamentales: poder asistir a un médico, tener una familia y un colegio donde aprender; o se elabora un mural con las manos de niños y niñas impresas incluyendo sus derechos, situándolo en un espacio escolar de transición entre zonas; o también se aprovechan las ramas de un árbol que ha sido pintado en el aula para tal ocasión con la finalidad de situar en ellas los diferentes Derechos.

Otra forma de celebración consiste en la proyección de un vídeo sobre los Derechos, conocer una canción sobre la temática tratando diferentes derechos cada día de la semana (esta opción fue propuesta por una alumna del Grado), fundamentalmente con dibujos y coloreando fichas. Se han dado situaciones en las que nuestro alumnado ha tenido que mostrar sus competencias docentes, bien porque su tutora de prácticas se encontraba de baja, bien porque esta no había planificado actividades para tal ocasión. La intervención del alumnado del Grado dio muestra de un gran sentido de responsabilidad, poniendo en marcha propuestas aprendidas en la universidad sobre la celebración del día de la Convención como fueron las relacionadas con la historia de los derechos de los niños usando las viñetas de Tonucci y la elaboración de una tarta conmemorativa que despertó la atención de las criaturas al cantar el 30 cumpleaños, dándose así paso a la explicación de dicha celebración.

Como conclusión más extendida, después del ejercicio de observación participante recogida en los informes y teniendo en cuenta el aprendizaje adquirido en la asignatura, el alumnado universitario interpreta mayoritariamente que estas actividades adolecen de cierta superficialidad. En un caso, se comenta que, con estas actividades puntuales, pasados unos días desde su celebración se comprobaba que el alumnado de Infantil se olvidaba de todo lo que habían realizado. Además, se resalta la diferencia de atención prestada por el profesorado a la hora de planificar actividades para el día 6 de diciembre, día de la Constitución. Como dato a 
destacar, en los 124 informes se hace mención a las actividades para la celebración del referéndum del día 6 de diciembre en escuelas y aulas donde se desarrolló del Practicum II.

\section{Discusión y conclusiones}

Los resultados obtenidos de los informes de investigación hechos por el estudiantado universitario como sujetos epistémicos (Rivero y Porlán, 2017) nos impulsan a seguir trabajando los contenidos de la Convención en la formación inicial. Hemos comprobado en esta investigación etnográfica que, aunque se desarrolle la celebración de forma mayoritaria en las aulas de Infantil en un $62 \%$ de los centros educativos, se detectan diferencias sustanciales en cuanto al espacio concedido a la CDN de 1989 en las escuelas observadas si se compara con otras celebraciones reconocidas socialmente, como fue el caso del Día de la Constitución de 1978. Estamos de acuerdo con las conclusiones a las que llegaron Parra y Segarra (2012) para Primaria, pues el modelo de educación para una ciudadanía democrática desarrollado en los espacios áulicos de Infantil es de perfil bajo. Este aspecto enlaza con lo defendido por Novella y Trilla (2014) cuando denuncian el predominio de una participación infantil más ficticia que real. En esta línea reivindicativa, entendemos que una de las finalidades de la escuela pública consiste en fomentar una lectura crítica y transformadora del entramado social para promover una intervención de la ciudadanía infantil en su medio. En consonancia con lo expresado por Ayuste y Trilla (2020), las instituciones educativas tienen la oportunidad de convertirse en espacios públicos de reflexión crítica sobre las cuestiones de poder entre diferentes grupos etarios. Pensamos que es necesario, desde la función docente, dar un decidido paso cultural y político para fortalecer la democracia real en un escenario de intermediación donde el profesorado ha de tomar partido. Se evidencia pues la imposible neutralidad del profesorado (Santisteban, 2019) ante las problemáticas generadas por la desigualdad social y política.

Una de las causas de esa circunstancia creemos que reside en la insuficiente formación y conciencia activa en el profesorado que debe acompañar a las criaturas a la hora de experimentar diariamente la práctica democrática y no sólo en los momentos de las celebraciones puntuales. En ese sentido, las conclusiones a las que ha llegado el estudiantado representan una prueba evidente para orientar la formación inicial. Como dice nuestro alumnado, sí, mayoritariamente se realizan actividades puntuales siendo estas superficiales. No obstante, se confirma que los y las infantes olvidan lo aprendido porque no han tenido la oportunidad de practicar diariamente el oficio de la ciudadanía (Bárcena, 1997) en la escuela y de un modo significativo. Esto se fundamenta en esa mirada que está muy arraigada en la mente colectiva pues entiende que este alumnado es muy pequeño e inmaduro para participar en las cuestiones políticas. Sin embargo, la investigación reciente nos indica que el contenido de las CC.SS. enseñadas y aprendidas en EI puede contribuir a la consolidación de una educación democrática y de justicia social (Gutiérrez y Pagès, 2018), superándose un conocimiento histórico escolar protagonizado por adultos, varones y con un carácter eminentemente etnocéntrico (Ortega, 2020). Por esa razón, la CDN de 1989 puede ser un excelente contenido para ser trabajado en Infantil a lo largo del curso escolar con el fin de visibilizar la contribución de las infancias a las sociedades democráticas en el marco de una ciudadanía global. 
Después de haber diseñado este proceso de formación inicial en sus distintas fases, la conclusión a la que llegamos es que un tratamiento amplio y bien planificado de los contenidos de la CDN por parte de los y las docentes en los centros escolares contribuiría enormemente al reconocimiento de los niños y niñas como protagonistas de una ciudadanía activa. Esta acción formativa puede repercutir en un cambio de mirada hacia las y los infantes, como sugieren Bruck y Ben-Arieh (2020), superando las resistencias del adultocentrismo (Gaitán, 2018). Se trata de un recurso imprescindible para desarrollar al máximo las diferentes capacidades de las criaturas de Infantil para ejercer sus derechos y deberes como parte integrante de la ciudadanía en la actual sociedad democrática (Urrea et al, 2018).

La realidad educativa explorada nos anima a seguir formando a un futuro profesorado novel para que sea consciente del valor histórico del contenido de la Convención y lo aplique regularmente en su desarrollo profesional. Estamos convencidos de que los derechos de la infancia no sólo se tienen que conocer en su celebración anual, sino que también han de vivirse y practicarse diariamente en los centros educativos, tal y como defienden Novella y Trilla (2014). Poseemos ya experiencias innovadoras e investigaciones (Urrea et al., 2018) que nos están indicando el camino a seguir. Es por eso por lo que, como escriben Porlán, Rivero y Martín del Pozo (1998), la formación inicial ha de incidir en los procesos de pensamiento, creencias y concepciones pedagógicas del futuro profesorado novel para interiorizar su función como agente de transformación social, contemplando este contenido de la CDN como un eje fundamental tanto en la Didáctica específica de las CC.SS. como en la construcción de la identidad docente de la etapa educativa de Infantil.

\section{Referencias bibliográficas}

Ayuste, A. y Trilla, J. (2020). Un sexto principio para el manifiesto por una pedagogía post-crítica. Teoría de la Educación, 32(2), 35-26.

Bárcena, F. (1997). El oficio de la ciudadanía. Introducción a la educación política. Barcelona: Paidós.

Bruck, S.; Ben-Arieh, A. (2020): La historia del estudio Children's Worlds, Sociedad e Infancias, (4), 35-42.

Carretero, M. (2019). Historia y conmemoraciones. Íber. Didáctica de las ciencias sociales, geografía e historia, (97), 4-7.

Estepa, J. y García Pérez, F.F. (2020). El modelo del profesor investigador en el aula en el Proyecto IRES. İber. Didáctica de las Ciencias Sociales, Geografía e Historia, (100), 22-28.

Foucault, M. (2013). El Poder, una bestia magnífica, sobre el poder, la prisión y la vida. Madrid: Editorial Siglo XXI

Gaitán, L. (2006). La nueva sociología de la infancia. Aportaciones de una mirada distinta. Política y Sociedad. 43 (1), 9-26.

Gaitán, L. (2018). Los derechos humanos de los niños: ciudadanía más allá de las “3Ps", Sociedad e Infancias, 2, pp. 17-37.

Galindo, R. (2016). Enseñar y aprender Ciencias Sociales en Educación Primaria. Modelo didáctico y estrategias metodológicas. En Ángel Liceras y Guadalupe Romero (Coords.), 
Didáctica de las Ciencias Sociales. Fundamentos, contextos y propuestas (pp. 73-93). Madrid: Pirámide.

Gutiérrez, M. C. y Pagès, J. (2018). Pensar para intervenir en la solución de las injusticias sociales. Pereira: Universidad Tecnológica de Pereira. Facultad de Ciencias de la Educación.

Hart, Roger A. (1993). La participación de los niños: de la participación simbólica a la participación auténtica, Innocenti Essay (4), International Child Development Centre.

Hoyuelos, A. y Riera, M.A. (2015). Complejidad y relaciones en educación infantil. Barcelona: Octaedro-Rosa Sensat.

Jerome, L., Emerson, L., Lundy, L., Orr, K. (2015). Teaching and learning about child rights: A study of implementation in 26 countries. Ginebra: UNICEF.

Lay-Lisboa, S. y Montañés, M. (2018). De la participación adultocéntrica a la disidente: La otra participación infantil. Psicoperspectivas. Individuo y Sociedad. 17 (2). http:// Doi: 10.5027/psicoperspectivas-Vol17-Issue2-fulltext-1176

Liebel, M. (2019). Janusz Korczak, los derechos y el protagonismo de la infancia. RES, Revista de Educación Social, (28), 175-195.

López, M. L., Felices, M. M., Giménez, M. D. y Moreno, C. (2018). El Practicum como estrategia formativa del pensamiento histórico en estudiantes de los Grados en Educación Infantil y Primaria. En E. López Torres, C. R. García Ruiz y M. Sánchez Agustí (Eds.). Buscando formas de enseñar. Investigar para innovar en DCS (pp. 243-252). Valladolid: Ediciones Universidad de Valladolid y AUPDCS.

Llena, A. y Novella, A. (Coords.) (2018). Impulsar la participación infantil. Los consejos de infancia y adolescencia. Barcelona: Graó.

Morin, E. (2001). Los siete saberes necesarios para la educación del futuro. Barcelona: Paidós.

Novella, A. M. y Trilla, J. (2014). La participación infantil. En Ana María Novella et al, Participación infantil y construcción de la ciudadanía (pp. 13-28). Barcelona: Graó.

Ortega, D. (2020). Problemas sociales, identidades excluidas y género en la enseñanza de la Historia. Lección inaugural del curso académico 2020-21. https://www.ubu.es/sites/default/files/portal_page/files/leccion_inaugural_2020-21.pdf

Pagès, J. (2019). Lliçó magistral, Joan Pagès i Blanch. Els reptes de l'ensenyament de les ciències socials, la geografia i la història. Quin professorat, quina ciutadania, quin futur? En M. Ballbé, N. González-Monfort, \& A. Santisteban (Eds.), Quin professorat, quina ciutadania, quin futur? Els reptes de l'ensenyament de les ciències socials, la geografia i la historia (pp. 11-35). Barcelona: Universitat Autònoma de Barcelona.

Parra, D. y Segarra, J.R. (2012). Celebraciones escolares, ¿fiestas cívicas? El tratamiento escolar del 9 d'Octubre y el día de la Constitución en las aulas valencianas de Educación Primaria. Didáctica de las Ciencias Experimentales y Sociales, (26), 19-34.

Pérez, E., Baeza, Mํㅡ C. y Miralles, P. (2008). El rincón de los tiempos. Un palacio en el aula de educación infantil. Revista Iberoamericana de Educación, (48), 1-15.

Pérez Garzón, J. S. (2008). ¿Por qué enseñamos geografía e historia? ¿Es tarea educativa la construcción de identidades? Historia de la educación. Revista Interuniversitaria, (27), 3755. 
Porlán, R; Rivero, A. y Martín del Pozo, R. (1998). Conocimiento profesional y epistemología de los profesores I: Teoría, métodos e instrumentos. Enseñanza de las Ciencias, 15 (2), 155171.

Riera, Mà. A. (2015). Del mirar al observar. En Alfredo Hoyuelos y María A. Riera, Complejidad y relaciones en educación infantil, (pp. 55-88). Barcelona: Octaedro-Rosa Sensat.

Rivero, A. y Porlán, R. (2017). La evaluación en la enseñanza universitaria. En R. Porlán (Coord.), Enseñanza universitaria. Cómo mejorarla (pp. 73-91). Madrid: Ediciones Morata.

Rodríguez, M.A. y Claudino, S. (Coords.) (2018). ¡Nosotros proponemos! Ciudadanía, sostenibilidad e innovación geográfica ante los desafíos educativos de la sociedad. Barcelona: Graò

Sanz Gimeno, A. (2020): Situación demográfica de la infancia y la adolescencia actual. Una visión mundial, Sociedad e Infancias, (4), 5-21.

Santisteban, A. (2019). La práctica de enseñar a enseñar ciencias sociales. Hortas, M. J. y Dias, A. (coords.). Enseñar y aprender didáctica de las Ciencias Sociales: la formación del profesorado desde una perspectiva sociocrítica (pp. 1079-1092). Lisboa: Escola Superior de Educaçao de Lisboa, Asociación Universitaria de Profesorado de Didáctica de las Ciencias Sociales.

Tonucci, F. (2009). ¿Se puede enseñar la participación? ¿Se puede enseñar la democracia? Investigación en la Escuela, (68), 11-24.

UNICEF (2019). Guía para la obtención del reconocimiento como centro referente en educación en derechos de infancia y ciudadanía global. Bases de la convocatoria 2019. https://www.unicef.es/sites/unicef.es/files/unicef-educa-EED-basesconvocatoria-reconocimientos-2019-.pdf

Urrea, A.; Coiduras, J.; Alsinet, C.; Balsells, M르.; Guadix, I., y Belmonte, O. (2018). Derechos de la Infancia y Ciudadanía Global: una aproximación desde la comunidad educativa. Sociedad e Infancias, (2), 127-146. 